\title{
Gustos y preferencias de los radioescuchas en Acámbaro, Gto.
}

\author{
Tastes and preferences of radio listeners in \\ Acámbaro, Guanajuato.
}

Universidad Politécnica de Guanajuato

Open Access bajo la licencia CC BY-NC-ND (http://creativecommons.org/licenses/by-nc-nd/4.0/).

Autor: M. M. Mayra Verónica Barrera Figueroa Ocupación actual: Profesor de Tiempo Completo en la Unidad Académica del Sureste, extensión de la Universidad Tecnológica de León.
FORMACIÓN ACADÉMICA

2013-2015 Maestría en Mercadotecnia. (Carta de pasante y Diploma)

2002-2007 Licenciatura en Mercadotecnia, en La Facultad de Ciencias Administrativas Universidad de Guanajuato (certificado obtenido y Título). 


\section{RESUMEN}

En la ciudad de Acámbaro; Gto., se llevó a cabo una investigación cuantitativa, derivada de un análisis mixto secuencial, como parte de la primera fase de un benchmarking ${ }^{1}$; donde se recolectó información para detectar la principal radio que prefieren en la localidad. Este primer paso no se podía omitir; ya que a partir de los datos arrojados, se dará continuidad a una segunda fase (cualitativa ${ }^{2}$ ), con el fin de llevar a cabo un análisis minucioso; entre la competencia ${ }^{3}$ detectada y una organización de radio en específico (la cual no se menciona por cuestiones éticas); de ello se pretende generar estrategias mercadológicas y estás en la implementación, logren un aumento de clientes, los cuales son radioescuchas y anunciantes.

\section{Palabras Clave: Competencia directa, estrategias mercadológicas y benchmarking.}

\section{ABSTRACT}

In Acambaro, Gto City; a quantitative research was conducted, thanks and caused by a sequential mixed analysis, like part of the first 'benchmarking' phase; where was obtained necessary information in order to detect the main radio station that the city prefers. We cannot skip this first step; because with the obtained data, we still giving continuity to the second phase (qualitative), the purpose it's to do a thorough analysis; between detected competition vs specific radio organization (the which that was not mentioned by ethical reasons); with that, the intention it's to generate marketing strategies and apply them and get a customer's increasement, and they are listeners and sponsors.

\section{Keys words: Direct competition, marketing strategies and benchmarking.}

\section{INTRODUCCIÓN}

El propósito de este artículo es mostrar los resultados de una investigación mixta secuencial, sobre la preferencia de los radioescuchas que habitan en la ciudad de Acámbaro; Gto. Dicho análisis es el resultado de la primer fase de un proyecto de benchmarking, para una radio de la localidad antes mencionada; la cual por cuestiones éticas y basándose en la American Psychological Association (APA), no permite hacer mención del nombre real; por lo tanto en el presente se alude a un nombre ficticio, este será Radio Bajío.

Esta primera fase del proyecto se guio por medio de una investigación cuantitativa; porque mide fenómenos, utiliza estadística, prueba hipótesis y replica (Hernández, 2010); y dentro del desarrollo se podrá apreciar el proceso realizado.

Cabe señalar que el análisis se realizó también bajo una línea de investigación de "Administración y Desarrollo Emprendedor"; ya que derivado de los resultados, se llevará en una segunda fase un benchmarking solicitado por la Radio Bajío, en donde ellos generarán nuevos productos; como programación y estrategias mercadológicas para que aumenten sus radioescuchas y anunciantes; los cuales son sus principales clientes.

Además para la realización de la primera fase del proyecto se partió de que el benchmarking es un término inglés que se utiliza para referirse a una técnica de gestión empresarial, desarrollada por la empresa Rank Xerox, por la que la organización comparará sus niveles de eficiencia, con los estándares externos y ciertas prácticas internas propias para emular y corregir los posibles desfases existentes (Santesmases, Sánchez, \& Valderrey, 2003); por ello fue necesario detectar la competencia percibida por las personas que en realidad tienen el gusto por escuchar radio; para que una vez que se lleve a cabo el análisis y la empresa radiofónica comience a realizar sus gestiones de mejora.

Aunado al termino mencionado anteriormente los autores Wells, Moriarty y Burnett (2007) enfocan al benchmarking como "un esfuerzo comparable para predecir una meta lógica"; además mencionan que

1 Proceso de comparar los productos y los procesos de la compañía con los de la competencia o con los de las compañias líderes de otras industrias, a fin de encontrar formas de mejorar la calidad y el desempeño, lo cual es un término a fin con la organización radiofónica (Philip Kotler, 2011).

2 La investigación cualitativa tiene como eje fundamental el profundo discernimiento del proceder humano y de los motivos que lo rigen (Bautista, 2011).

3 La competencia es el conjunto de empresas que ofrecen productos iguales o similares a los de otra empresa o bien que hacen productos que aunque no sea igual puede sustituir el consumo de los de la otra empresa (Equipo Vértice, 2012) 
"se usa para identificar bases de auditorías previstas o auditorías de otras empresas e industrias relacionadas, para que haya un punto de comparación" (Wells \& Burnett, 2007); por lo tanto en primer instancia el termino empatizo con los objetivos a seguir; debido a que fue necesario conocer la preferencia que tiene la población acambarense, sobre las diferentes emisoras de radio que escuchan en la región; así mismo con los resultados obtenidos comenzar un análisis minucioso de lo que ofrecen las organizaciones radiofónicas y en paralelo; analizar que está ofreciendo en realidad Radio Bajío.

Finalmente se mostrará la aceptación o rechazo de las hipótesis ${ }^{4}$, basadas en la experiencia de la gerente general de la radio, sobre la principal competencia de la Radio Bajío.

\section{DETECCIÓN DE LA NECESIDAD}

La Radio Bajío en el año 2014, con el apoyo de una alumna perteneciente a la Unidad Académica del Sureste (UAS) llevó a cabo un análisis FODA ${ }^{5}$, el cual arrojó que la percepción de la misma era mala, debido a que la percibían como anticuada, derivado a que las voces que se escuchan en las estaciones tienen muchos años al aire novedosa; paralelo a ello, habían detectado bajas ventas, tanto en anunciantes como en radioescuchas. A partir de estos datos, la gerente general se percató de lo que realmente estaba ocurriendo, para ello necesitaban comenzar a realizar algo con el fin de mejorar.

Por ende, se eligió realizar un benchmarking para detectar qué ofrecía la competencia que ellos no están ofertando, pero no solo copiar si no innovar; para sustentar lo anterior, se basó en el criterio que aportó el sitio web el ciberconta.unizar.es; donde mencionan que:

"El benchmarking no se centra en copiar lo que los otros hacen bien. Evidentemente no siempre es asi o al menos, no debería serlo. Una empresa que lo aplique correctamente, debería identificar qué es lo que hacen bien otras empresas e intentar mejorar sus procedimientos (www.5campus.com, Recuperado el 15 de abril de 2015)".

4Explicaciones tentativas de la relación entre dos o más variables (Hernández, 2010)

5 Herramienta de provecho para la planificación estratégica, en particularpara lo que significa el análisis situacional para construir las estrategias del plan y modificarlas.(Hernández, 2011) Se forma del acrónimo Fortalezas, Oportunidades, Debilidades

\section{PREGUNTA DE INVESTIGACIÓN}

En este apartado se muestra el planteamiento de las preguntas de investigación, las cuales orientarán hacia las respuestas que se buscan con la investigación (Hernández, 2010), estas fueron la pauta para enfocar los esfuerzos y encontrar la competencia de la Radio Bajío.

Además la pregunta de investigación determinó el diseño de investigación y estableció qué resultados cabe esperar (Heinemann, 2003).

Las preguntas planteadas se muestran a continuación:

- ¿Cuál es la competencia de la radio local de Acámbaro? (Primera fase del proyecto).

- ¿Qué estrategias podrían ser aplicables a la Organización Radiofónica de Acámbaro? (Segunda fase del proyecto).

\section{JUSTIFICACIÓN DE ESTUDIO}

La justificación permite decir, entre otras cosas, por qué, nuestro problema es sustantivo y real, por qué lo hemos planeado, por qué es importante investigarlo y para qué va a servir hacerlo (Hurtado \& Josefina, 2007). Por lo tanto la justificación para esta investigación fue que el estudio se pretendió fuera rico de información debido al enfoque mixto secuencial, en donde se representaron un conjunto de procesos sistemáticos, empíricos y críticos de investigación e implican la recolección y el análisis de datos cuantitativos y cualitativos (Hernández, 2010).

La utilización de un benchmarking radica en que ofrece un contexto de comparación inmediato, es decir desde el momento que se está recopilando la información; según Sánchez (2008) brinda un panorama para determinar si la forma en la que se desarrollan las propias actividades y funciones representan una "mejor práctica" en la competencia.

Además la realización de un benchmarking, ayudará a la Radio del Bajío, para detectar algunas herramientas estratégicas que la competencia implementa; con la finalidad de captar más clientes. Así la empresa podrá mejorar las estrategias que arroje el análisis.

La investigación planteada contribuirá a detectar la competencia directa de la radiodifusora, para culminar con la primera fase del proyecto; y con ello pasar a la segunda fase de análisis cualitativo; que ayude a generar clientes, tanto radioescuchas como anunciantes; además de crear procesos que ayuden a brindar un servicio de calidad. 


\section{VIABILIDAD DEL PROYECTO}

El proyecto a realizar tuvo viabilidad ${ }^{6}$; debido a que se contó con el recurso humano (alumnos de la UAS), los cuales realizaron el levantamiento de la información; aunado de que no implicó un alto costo la recolección de datos, esto porque no se invirtió en material; ya que la UAS proporcionó el material necesario.

\section{OBJETIVOS DE LA INVESTIGACIÓN}

Se realizó la formulación de los objetivos ${ }^{7}$, para describir las perspectivas de la investigación y con ello especificar qué se espera de la investigación (Mohammad, 2005).

Los objetivos planteados para el "Análisis de preferencia de los radioescuchas en Acámbaro, Gto" fueron:

- Identificar la competencia de la Organización Radiofónica de Acámbaro, la cual es considerada por la población acambarense. (Primera fase).

- Detectar las estrategias que podrían ser aplicables a la organización radiofónica de Acámbaro. (Segunda fase).

\section{DETERMINAR EL TIPO DE INVESTIGACIÓN.}

Para poder dar respuesta a la pregunta de investigación, ¿Qué estrategias podrían ser aplicables a la Organización Radiofónica de Acámbaro? el tipo de investigación llevado a cabo fue mixto secuencial, con un componente cuantitativo y otro cualitativo; con el fin de encontrar una fotografía más completa del problema (Hernández, 2010)

Es secuencial, porque los resultados de la primera parte, la cuantitativa; servirá para desarrollar la segunda fase, la cualitativa.

\section{- $\quad$ PRIMER FASE CUANTITATIVA}

Reconocer la competencia directa.

Para la primera fase del proyecto el tipo de investigación fue cuantitativa con un diseño no experimental transversal; en donde se definió un alcance descriptivo. En primera instancia se utilizó cuantitativo, debido a que se requirió conocer la tendencia en cuanto a las radiodifusoras que considera la población acambarense; de su preferencia, las cuales se tomaron como la competencia directa de Radio Bajío.

El diseño fue no experimental transversal; ya que los datos se recolectaron en un solo tiempo; aunado a lo anterior el propósito fue describir su incidencia en los habitantes de la zona centro de Acámbaro, es decir que se basó en los autores Toro Iván y Parra Rubén; los cuales en su libro "Método y conocimiento, metodología de la investigación" mencionan que una investigación no experimental es observar fenómenostal y como se dan en su contexto natural, para después analizarlos (Toro \& Parra, 2006).

Asimismo fue una investigación descriptiva; porque se detalló la competencia, y por medio de ese estudio, se buscó especificar propiedades, características y rasgos importantes (Hernández, 2010) en este caso de las diferentes estaciones de radio a analizar, ya que servirán para la segunda fase y serán el punto de comparación para Radio Bajío.

\section{ESTABLECERFUENTESDEINFORMACIÓN}

\section{- Fuentes primarias internas}

Las fuentes primarias son aquellas que deben ser elaboradas por el investigador, debido a que no están disponibles (Merino María, Joaquín, \& Grande, 2010) Al comenzar a buscar información, en primera instancia se recurrió a las primarias internas; en donde se llevó a cabo una reunión con la gerente general con la finalidad de conocer acerca de la Radio Bajío, así como conocer sobre la organización radiofónica y con ello se pudo formular la investigación.

En esta reunión se habló acerca de la historia, esfuerzos de comunicación, metas de la empresa, clientes, servicio ofrecido e información general que han llevado a cabo dentro de sus estrategias comerciales; esto desde sus inicios hasta la fecha.

Dentro de esta etapa de la investigación, se procedió a elaborar un instrumento de recolección de datos, encuesta.

- $\quad$ Fuentes secundarias

Las fuentes secundarias, son aquellas que ya están procesadas y elaboradas previamente, por personas o entidades ajenas a la misma. (Merino María, Joaquín, \& Grande, 2010)

Otra fuente importante de información fueron las secundarias, en donde se revisaron libros e internet; estos datos sirvieron para sustentar el fundamento teórico y el tipo de investigación a seguir. 


\section{DEFINIR HERRAMIENTAS RECOLECCIÓN DE DATOS.}

La herramienta que se utilizó para recolectar datos fue la encuesta; ya que recoge información proporcionada de verbalmente o por un informante mediante un cuestionario estructurado (Alvira, 2011), se tuvo que tener una estructura para no desviar la atención del objetivo de conocer competencia directa de la Radio Bajío.

Para la elaboración de la encuesta, se tuvo que comenzar realizando un check list ${ }^{8}$ de tópicos; para así mismo, realizar preguntas adecuadas y que el radioescucha expresará el nombre de la estación que escucha con mayor frecuencia.

Los tópicos que se determinaron fueron:

- $\quad$ Gusto por la radio

- Frecuencia de escucha

- Formato para escuchar la radio

- $\quad$ Estación de preferencia

- Programación

- $\quad$ Medio por el cual escuchan la radio

- $\quad$ Recomendaciones de contenido.

- $\quad$ Preferencia de temática para un programa

- $\quad$ Recomendaciones de mejora

Una vez con la lista de puntos que se deseaba conocer se realizó la encuesta definitiva. En seguida se muestran las preguntas realizadas, con sus respectivas opciones: 1.- ¿Le gusta la radio?
A) $\mathrm{SI}$
POR QUÉ (Continúe la encuesta)
B) $\quad \mathrm{NO}$
POR QUÉ (Pase a la pregunta 9)

2.- ¿Con qué frecuencia escucha la radio?
A) Todos los días
B) 1 vez a la semana
C) 2 veces por semana

3.- ¿Qué formato es de su preferencia?

A) Radio convencional

4.- ¿Cuál es su estación favorita?

5.- ¿Qué te ofrece la estación que mencionaste, para que sea su favorita?

6.- ¿En qué lugar escucha la radio?
A) Casa
B) Automóvil
C) Trabajo

7.- ¿Cuál es la sección del día para que la escuche?
DE B) Por la tarde

C) Por la noche

8.- ¿Qué tipo de programas escucha?

A) Informativos

B) Culturales

C) Deportivos

D) Musicales

E) Espectáculos

F) Interactivos

G) Otros

9.- Qué debería tener la radio para que la escuche

\section{HIPÓTESIS}

Las hipótesis son explicaciones supuestas que están bajo ciertos hechos, a los que sirve de soporte (Rodríguez, 2005); cabe mencionar que estás surgen de los objetivos planteados.

Para el proyecto realizado en la Radio Bajío, se planteó una hipótesis considerando algunas estaciones de radio que tienen frecuencia en la localidad; además que la gerente general emitió una estación, basándose en la experiencia que tiene laborando para la radio; está se menciona a continuación:

- Máxima y EXA, son las estaciones de mayor preferencia por las personas que transitaban por la zona centro de Acámbaro.

\section{MUESTRA}

La muestra es una parte representativa de la población. En el caso de la investigación cuantitativa, existen métodos probabilísticos ${ }^{9}$ y no probabilísticos ${ }^{10}$. Para conocer las radiodifusoras de preferencia de la población acambarense; se llevó a cabo una un método no probabilístico, específicamente un muestreo de conveniencia ${ }^{11}$, debido a que según el sitio oficial del Instituto Nacional de Geografía y Estadística (INEGI, 2010); Acámbaro cuenta con 109,030 habitantes; por lo tanto sería casi imposible encuestar a cada uno de ellos. Además la muestra por conveniencia apoyó, porque suele usarse en estudios iniciales para comprobar si se cumplen las hipótesis que se plantea el investigador (universoformulas.com, 2015, Recuperado el 17 de abril de 2015)

Finalmente se determinó que la muestra sería de 100 habitantes de Acámbaro; Gto., que transitaban por el jardín principal de la localidad; cabe señalar que esté se encuentra ubicado en la zona centro.

\section{A) Por la mañana}




\section{RECOLECCIÓN DE LA INFORMACIÓN}

La recolección de datos se llevó el día 20 de marzo de 2015, en la zona centro de Acámbaro, específicamente en el jardín principal; esto en el lapso de 10 de la mañana a 2 de la tarde. Los alumnos de la carrera de Desarrollo de Negocios, pertenecientes a la Unidad Académica del Sureste (UAS); participaron en el levantamiento de la información.

\section{ANÁLISIS E INTERPRETACIÓN DE RESULTADOS}

Los resultados de la encuesta aplicada a las personas que transitaban por el jardín principal de Acámbaro; servirán para tomar decisión de a qué estaciones de radio se aplicará el benchmarking (segunda fase del proyecto), a continuación se muestran los resultados:

\section{Pregunta 1.- ¿Le gusta escuchar la radio?}

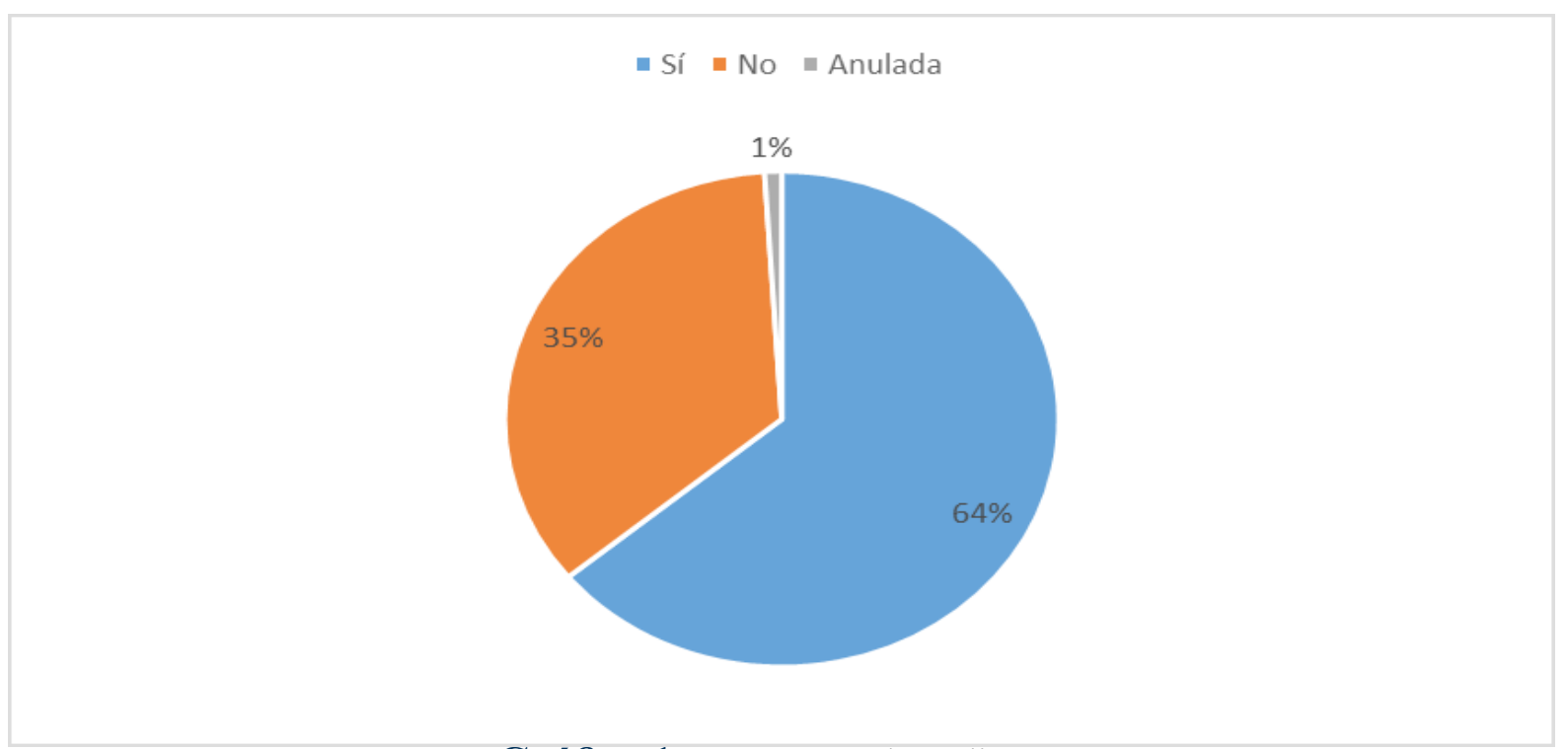

\section{Gráfica 1.- Gusto por la radio}

En la gráfica se aprecia la respuesta sobre el gusto por la radio, en donde a la mayoría de la población acambarense sí les gusta escuchar la radio; esto reflejado con un $64 \%$, sin embargo el $35 \%$ respondió que no le agradaba y, finalmente se observa un $1 \%$ el cual corresponde a una encuesta que fue anulada, esto debido a que no eran legibles las respuestas, se consideró ya que eso podría ocasionar un sesgo ${ }^{12}$.

9Muestreo probabilístico es un tipo de muestreo en el que se conoce la probabilidad de seleccionar un miembro individual de la población (Quintana, Cabañas, \& Gómez, 1998).

10 Muestreo no probabilístico es aquel en el que se desconoce la probabilidad de seleccionar cualquier miembro de la población (Quintana, Cabañas, \& Gómez, 1998).

11 Método de muestreo no probabilístico. Consiste en seleccionar a los individuos que convienen al investigador para la muestra. 12 Se refiere a errores sistémicos que afectan a cualquier muestra; representan la diferencia entre el

valor muestral esperado y el valor verdadero (Arnau \& Anguera, 1990) 


\section{Por qué sí}

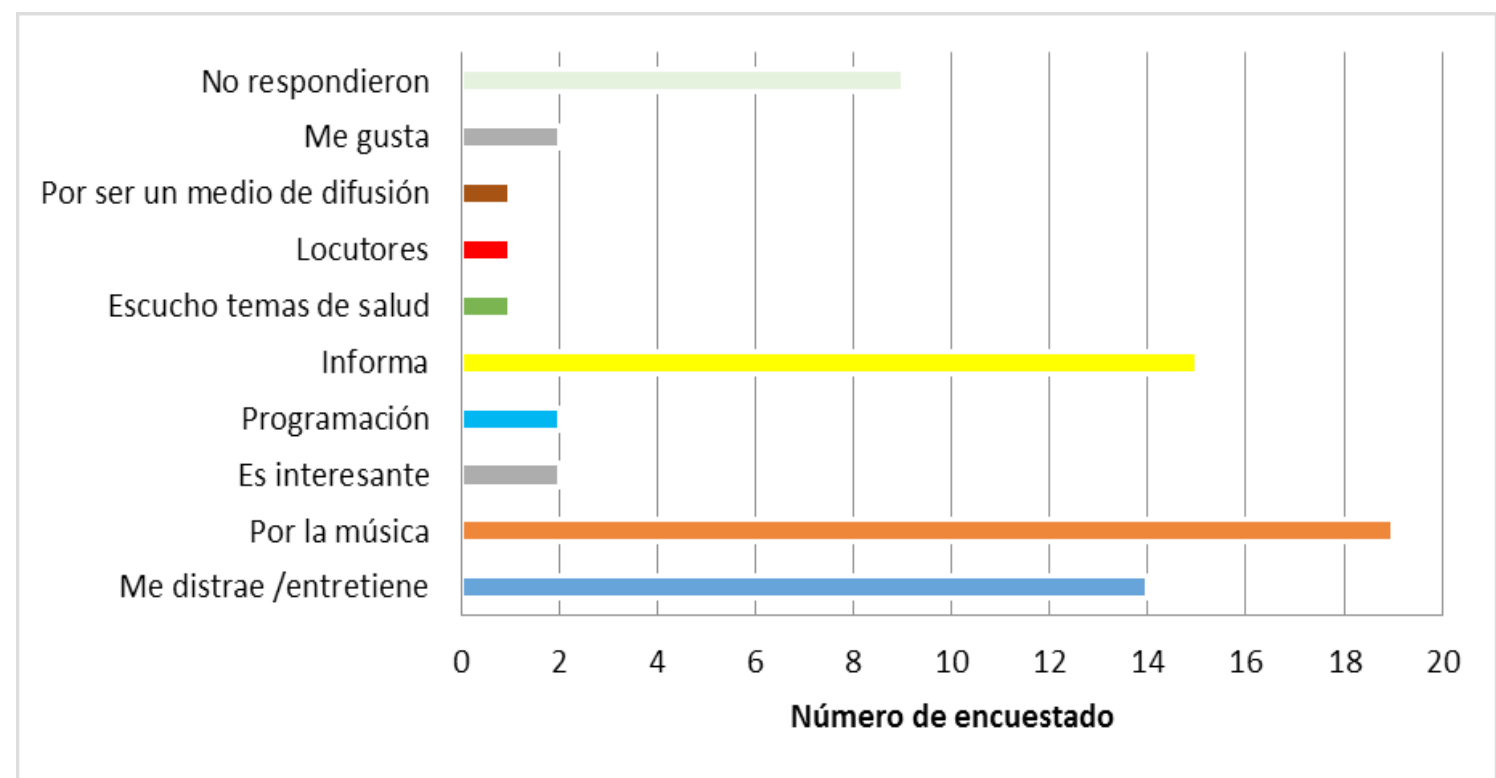

Gráfica 2.- Motivos por el cual escuchan la radio.

Dando seguimiento a la pregunta 1, en donde el $64 \%$ de los respondientes expresó sí gustarles la radio; se les cuestionó ¿por qué?, y en la gráfica 2 se aprecia que un 28.8\% (19 encuestados) opinó que le gustaba la radio por la música, así mismo el $22.7 \%$ (15 encuestados) dijo que era porque informa; sin embargo un 21.2\% (14 encuestados) expresó que la razón es que los distrae o entretiene, por otro lado el 13.6\% (9 encuestados) no dieron motivos por el gusto, es decir dejó en blanco el cuestionamiento. Posteriormente se encuentran los porcentajes más bajos, los cuales oscilan en 3\% y $1.5 \%$; estos se detallan en seguida: con un 3\% existieron tres respuestas diferentes, estás son: por la música (2 encuestados), otra fue que simplemente les gusta (2 encuestados); y por último debido a la programación (2 encuestados).

Finalmente el $1.5 \%$ dijo que por los locutores (1 encuestado), porque escuchan programas de salud (1 encuestado); y porque es un medio de comunicación (1 encuestado).

\section{ANÁLISIS E INTERPRETACIÓN DE RESULTADOS}

\section{Por qué no}

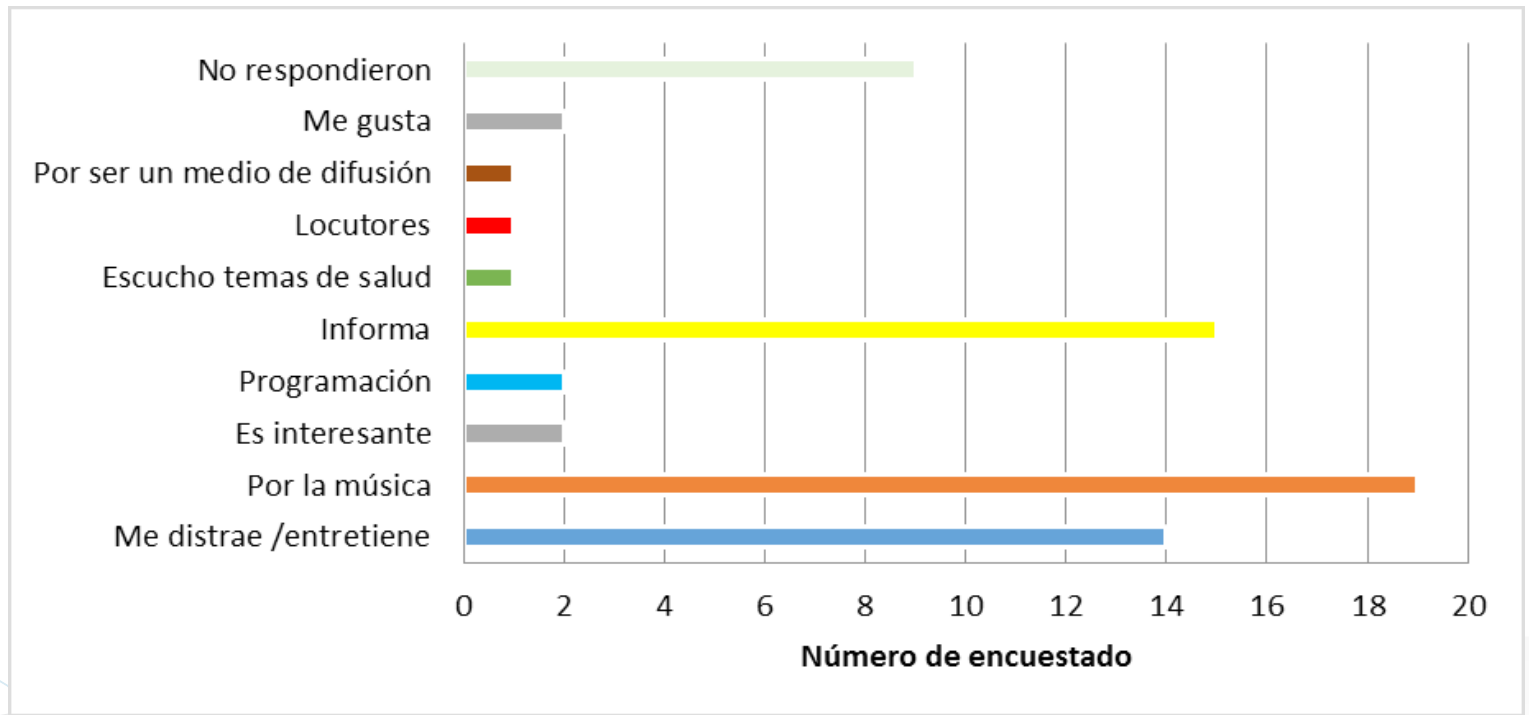

Gráfica 3.- Razones por las cuales no les gusta la radio. 
La gráfica 3 se deriva del $35 \%$ de la población que transitaba por la zona centro de Acámbaro, que respondieron no gustarles la radio. En la imagen se aprecia que la tendencia mayor fue un $28.6 \%$ (10 encuestado); la cual dijo no gustarle porque no tienen tiempo, sin embargo el $17.1 \%$ (6 encuestados) mencionó que es aburrida; así mismo le sigue el $8.6 \%$ (3ncuestados) que dijo no tener radio, de igual manera se observa que un $5.7 \%$ respondió no acostumbrarla y que no le llama la atención (2 encuestados).

Por otro lado se muestran varias respuestas de la población acambarense con $2.9 \%$ (1 encuestado diferente), estas son:

- $\quad$ No aprecian que haya un surtido en temas.

- Simplemente no la escuchan.

- Piensan que tratan temas informales.

- Prefieren ver la televisión.

- $\quad$ No les llega bien la frecuencia a su casa.

- $\quad$ Ponen cosas que no son música.

- Escuchan música en su celular.

- Consideran que existe exceso de comerciales.

- $\quad$ No respondieron, es decir dejaron en blanco el cuestionamiento.

\section{Pregunta 2.- ¿Con qué frecuencia escucha la radio?}

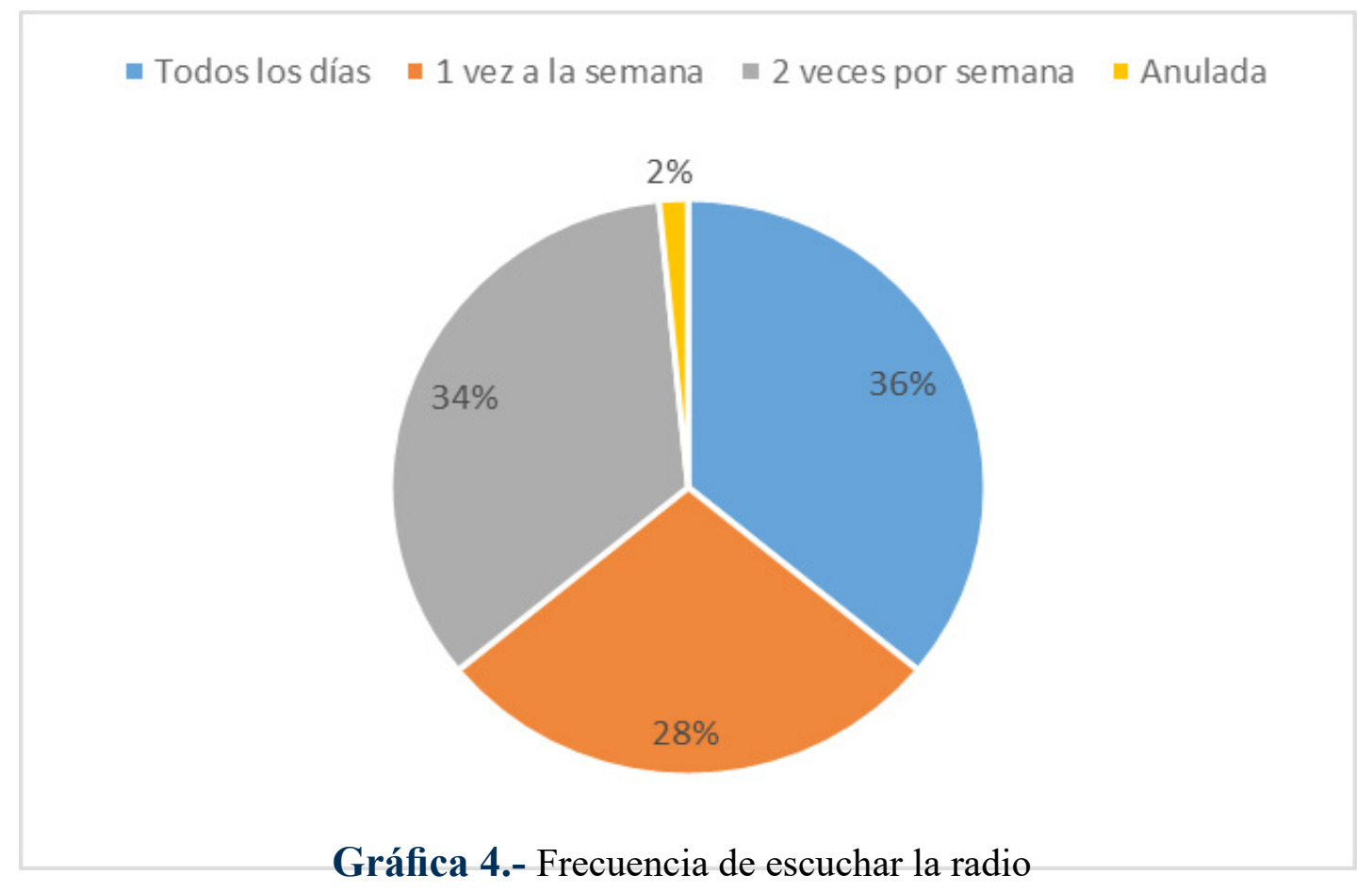

En la imagen se observa que la población encuestada en Acámbaro, respondió con qué frecuencia escucha la radio; en donde el $36 \%$ mencionó escucharla todos los días, sin embargo el $34 \%$ dijo 2 veces por semana; aunado al $28 \%$ que contestó 1 vez a la semana, y finalmente; el $2 \%$ pertenece a 1 encuesta anulada debido a que no eran legibles las respuestas. 


\section{ANÁLISIS E INTERPRETACIÓN DE RESULTADOS}

\section{Pregunta 3.- ¿Qué formato es de su preferencia?}

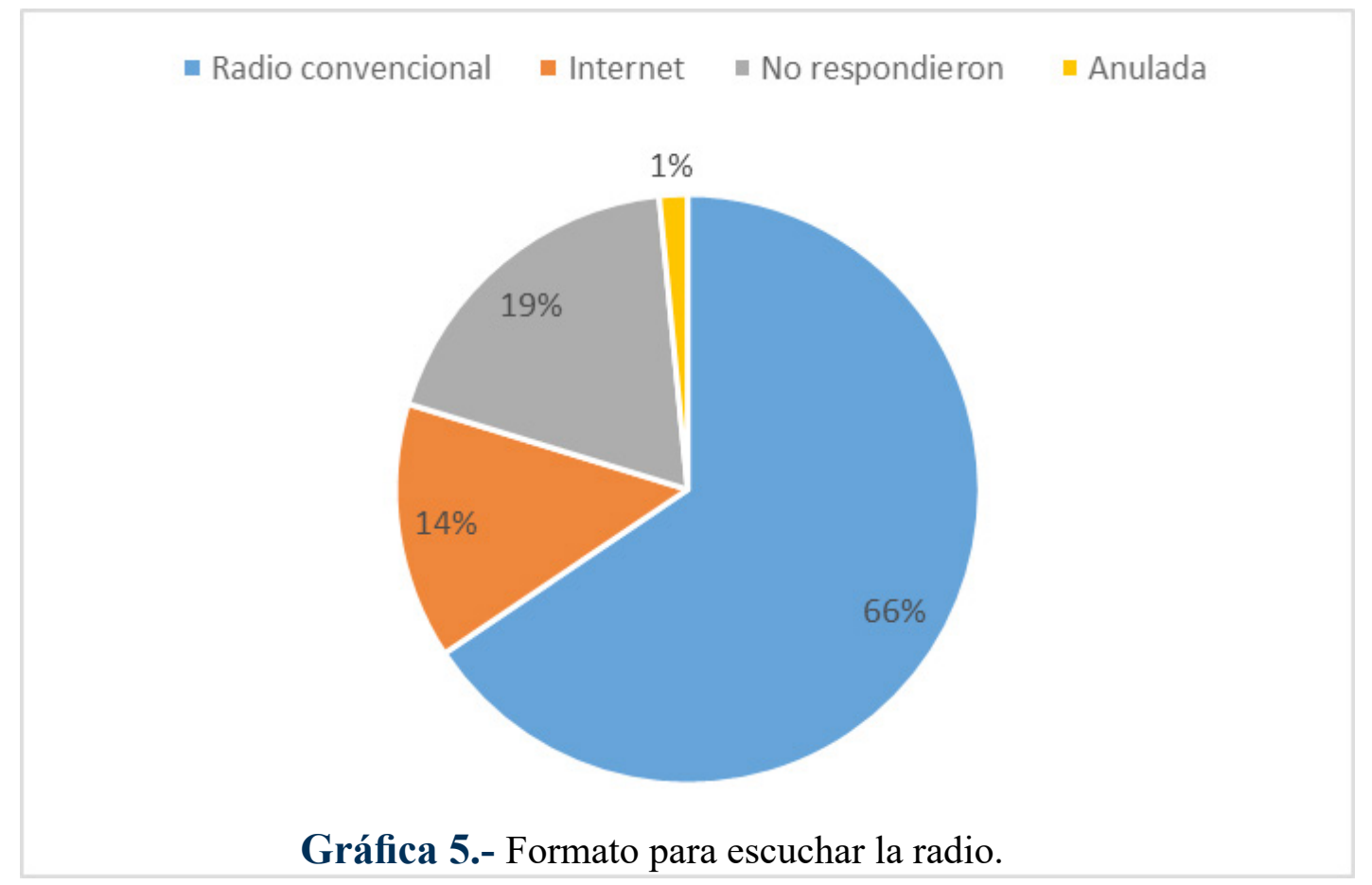

En la gráfica 5 se aprecia que la población acambarense respondió al cuestionamiento sobre el formato que usan para escuchar la radio; en donde el 66\% dijo que hacen uso de la radio convencional, por otro lado el 19\% no respondió, es decir dejo el cuestionamiento en blanco, así mismo el $14 \%$ contesto que utiliza el internet; y finalmente, un $1 \%$ el cual pertenece a una encuesta anulada porque los datos no eran legibles.

\section{Pregunta 4.- ¿Cuál es tu estación favorita?}

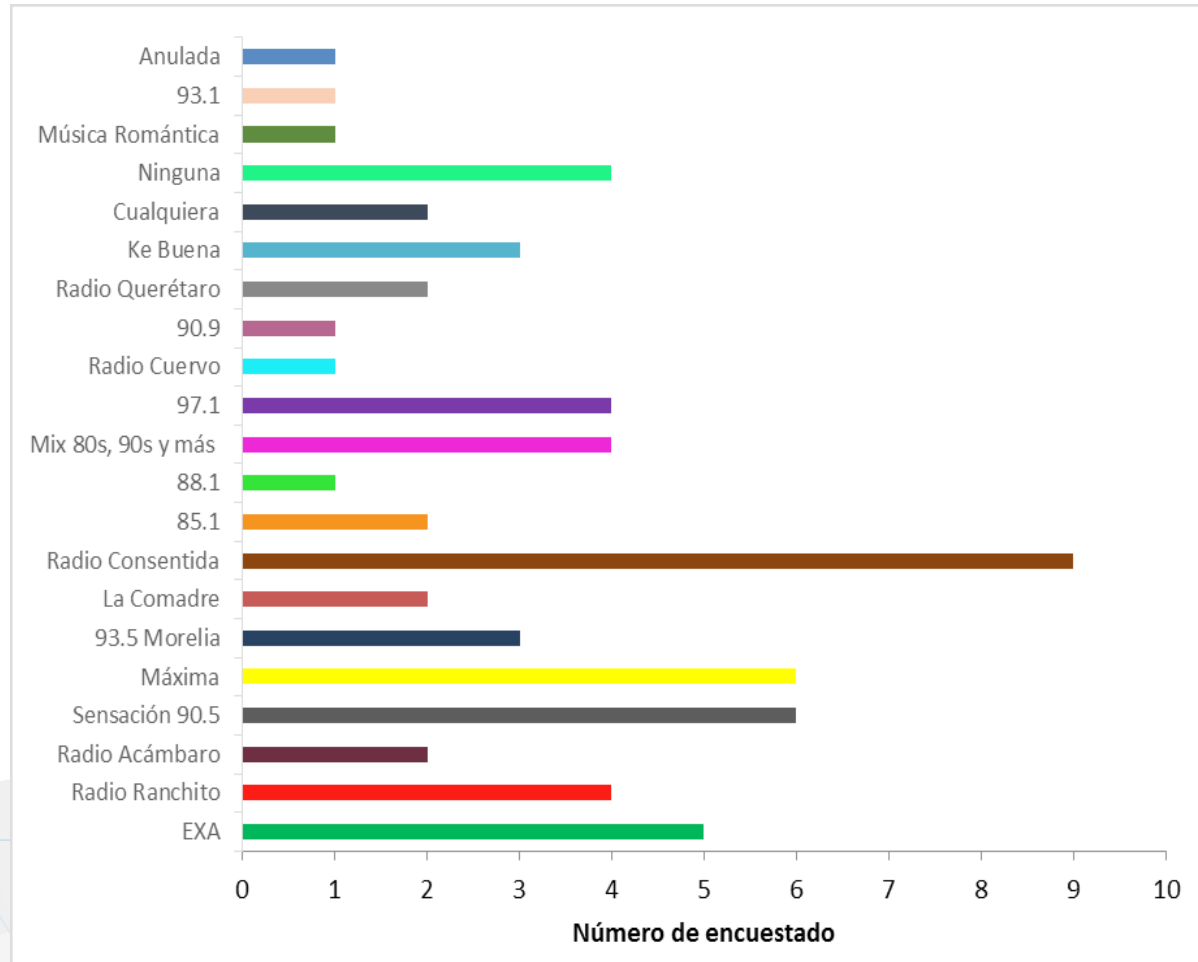


Gráfica 6.- Estación de preferencia.

La gráfica 6 muestra la tendencia sobre la estación favorita de las personas, que transitaban por el jardín principal situado en la zona centro de Acámbaro; las cuales respondieron que sí escuchan la radio, en donde se observa con un $14 \%$ que la estación de preferencia es "La consentida", así mismo se encuentra un empate con 9\% que dijeron "Sensación 90.5" y "Máxima", por otro lado el 8\% dijo "EXA", sin embargo se aprecian con un 6\% cuatro respuestas diferentes las cuales son: "Radio Ranchito", "Mix 90's, 80’s y más", “97.1 la Z” y que no tienen ninguna en específico.

Por otra parte se encuentra un 5\% que mencionó "93.5 de Morelia" y "Ke buena" eran sus estaciones favoritas; además se observan con 3\% varias respuestas como: "La Comadre", "Radio de Acámbaro" (en la cual no mencionaron el nombre en específico) "85.1” y
"Radio Querétaro" (donde de igual manera no especificaron el nombre) y mencionaron que cualquier estación estaba bien.

\section{ANÁLISIS E INTERPRETACIÓN DE RESULTADOS}

Finalmente los datos arrojaron 6 respuestas diferentes con un $2 \%$, las cuales se mencionan a continuación:
- $\quad 93.1$
- Música Romántica
- $\quad 90.9$
- $\quad$ Radio Cuervo
- $\quad 88.1$
- $\quad$ Encuesta anulada, debido a la poca legibilidad de datos.

\section{Pregunta 5.- ¿Qué te ofrece la estación que mencionaste, para que sea su favorita?}

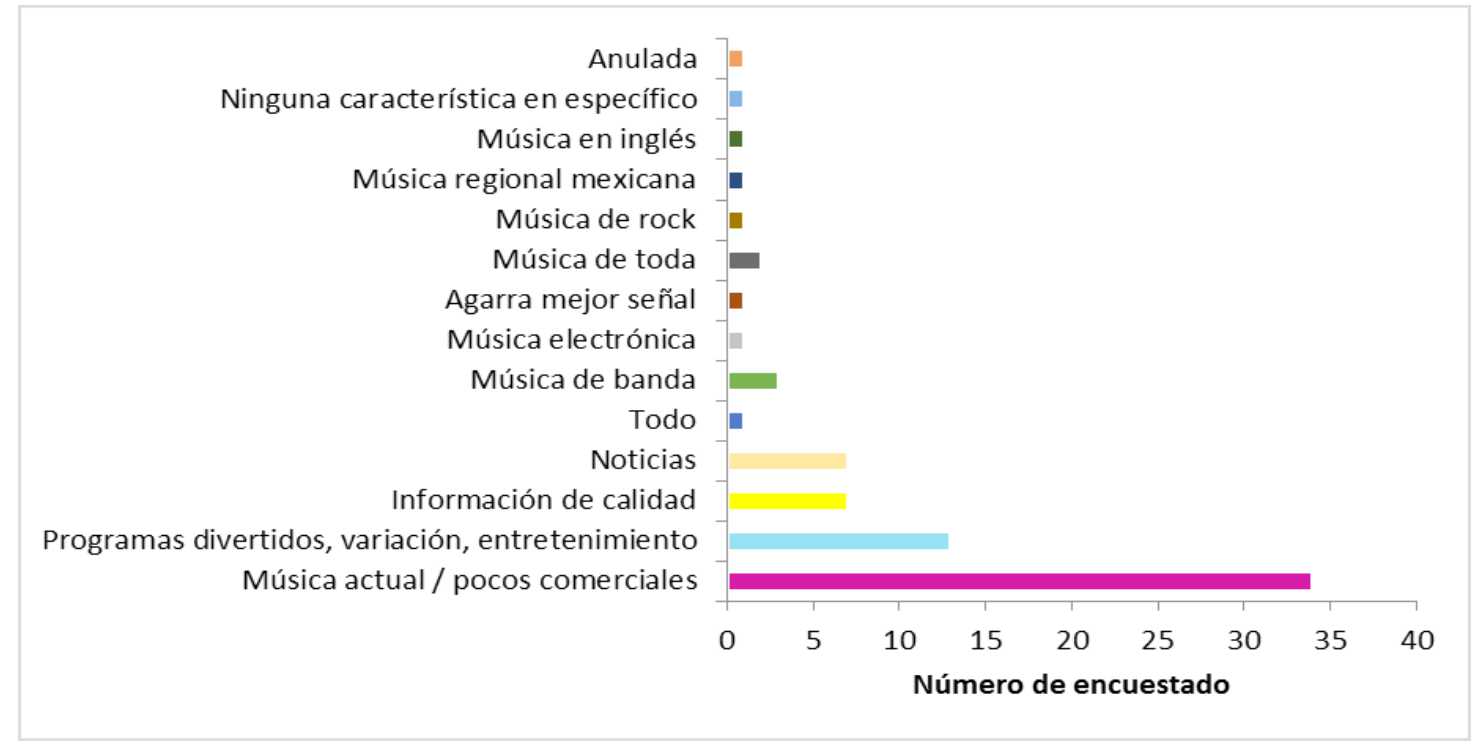

Gráfica 7.- Qué ofrece la estación de preferencia.

En la imagen se aprecian las tendencias de la población, sobre lo que ofrece la estación de su preferencia; siendo únicamente 74 personas que respondieron a este cuestionamiento. El 45.9\% (34 encuestados) mencionó les brindan música actual y pocos comerciales; por otro lado el 17.6\% (13 encuestados) respondió porque les ofrecían programas divertidos, variación de música y entretenimiento; sin embargo hubo un empate del 9.5\% (7 encuestados) que contestaron brindarles información de calidad y noticias.

Asimismo el 4.1\% (3 encuestados) expresó que le ofrecían música de banda, en cambio el $2.7 \%$ (2 encuestados)
Finalmente con el 1.4\% ( 1 encuestado diferente) se observan varias respuestas, las cuales se mencionan a continuación:

- $\quad$ Le ofrecen de todo.

- Música en inglés.

- Música regional mexicana.

- Música rock.

- Mejor señal.

- Música electrónica.

- Ninguna característica en específico.

- $\quad$ Encuesta anulada, debido a la legibilidad de los datos. 
Pregunta 6.- ¿En qué lugar escucha la radio?

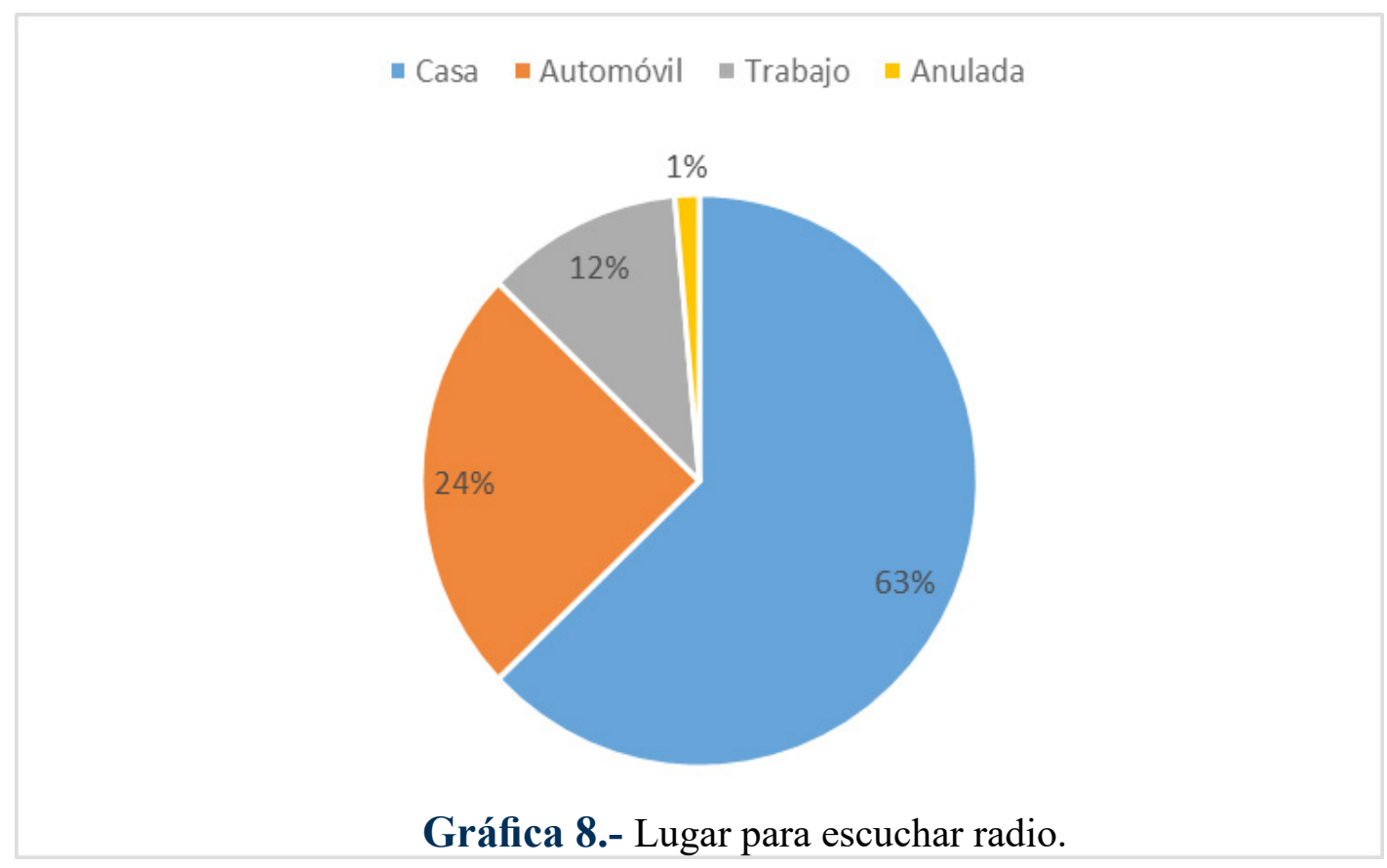

Gráfica 8.- Lugar para escuchar radio.

En la gráfica 8, se aprecia en qué lugar la población encuestada en el jardín principal de Acámbaro escucha la radio; en donde el $63 \%$ respondió escucharla en la casa, sin embargo el $24 \%$ dijo en el automóvil; en cambio el $12 \%$ mencionó en el trabajo y finalmente; el $1 \%$ pertenece a una encuesta anulada, debido a que no eran legibles los datos.

\section{ANÁLISIS E INTERPRETACIÓN DE RESULTADOS}

\section{Pregunta 7.- ¿Cuál es la sección del día para que la escuche?}

$$
\text { - Mañana - Tarde } \| \text { Noche } \| \text { Anulada }
$$

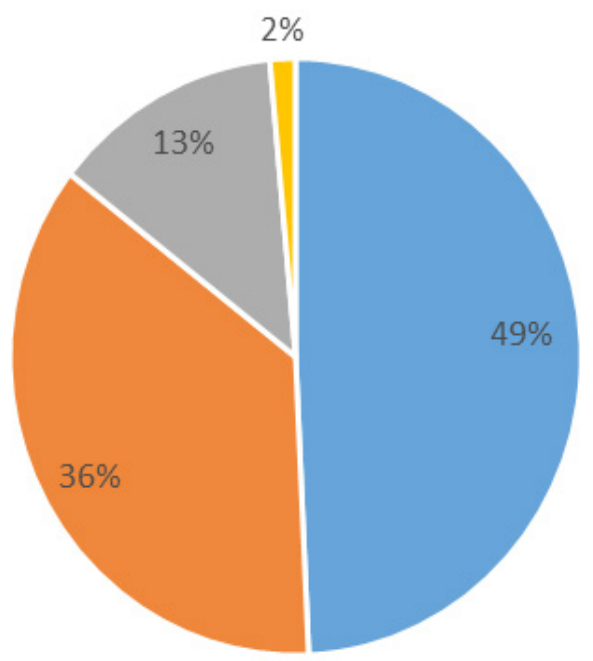

Gráfica 9.- Momento del día para escuchar radio. 
En la imagen se observa la tendencia sobre el momento del día, en que hacen uso de la radio; las cuales fueron respuestas brindadas por la población acambarense que transitaban por el jardín principal de la ciudad, en donde el $49 \%$ mencionó que por la mañana prefieren escucharla; sin embargo el $36 \%$ dijo por la tarde y el $13 \%$ eligió por la noche. Cabe señalar que el $2 \%$ pertenece a una encuesta que fue anulada debido a que los datos eran poco legibles.

\section{Pregunta 8.- ¿Qué tipo de programas escucha?}

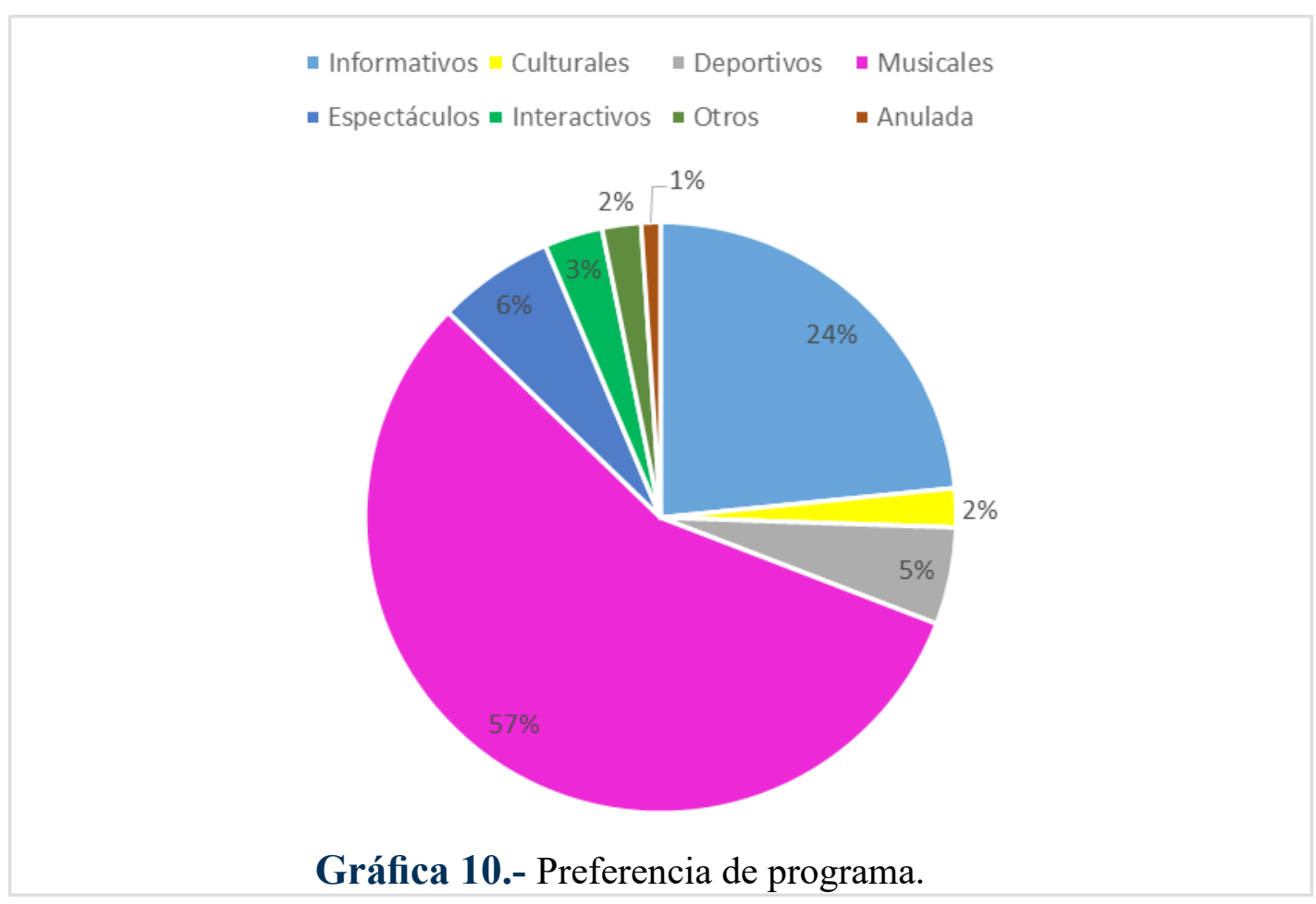

En la gráfica 10 se observa el tipo de programas que prefiere la gente de Acámbaro, los cuales respondieron sí les gustaba la radio, en donde el 57\% mencionó que les agradan los musicales, en cambio el $24 \%$ prefieren los informativos; sin embargo el 6\% eligió programas de espectáculos, aunado a un 3\% que determinó los interactivos; y un 2\% eligió otros sin hacer mención del tipo de programa en específico.

Finalmente el 1\% pertenece a una encuesta anulada debido a la falta de legibilidad de los datos. 


\title{
ANÁLISIS E INTERPRETACIÓN DE RESULTADOS
}

\section{Pregunta 9.- Qué debería tener la radio para que la}

\author{
- Brindo recomendaciones \\ - No brindo recomendaciones
}

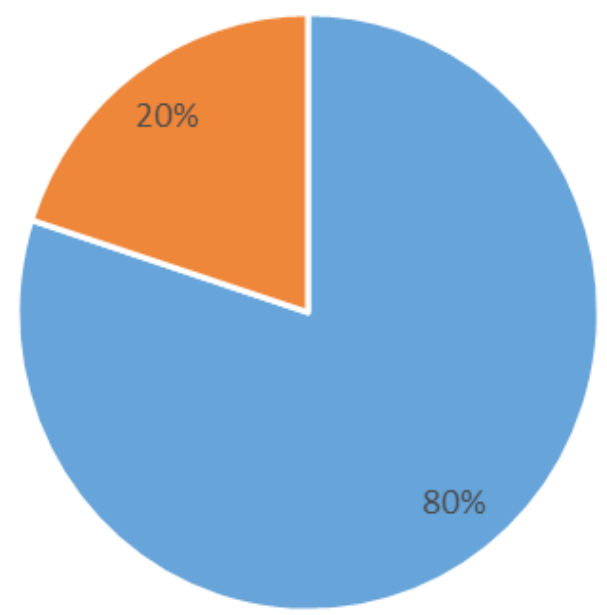

Gráfica 11.- Porcentaje de respuesta

Antes de interpretar los comentarios realizados por la población de Acámbaro, fue necesario saber qué porcentaje hizo aportaciones; y cuántos se negaron a responder.

En la gráfica se aprecia el índice de respuesta que emitió la población acambarense en la zona centro de la ciudad; en donde el 80\% sí brindo recomendaciones de mejora para que con ellas, tomará la decisión de escuchar la radio; en cambio el $20 \%$ no aporto a la pregunta dejando en blanco el cuestionamiento.

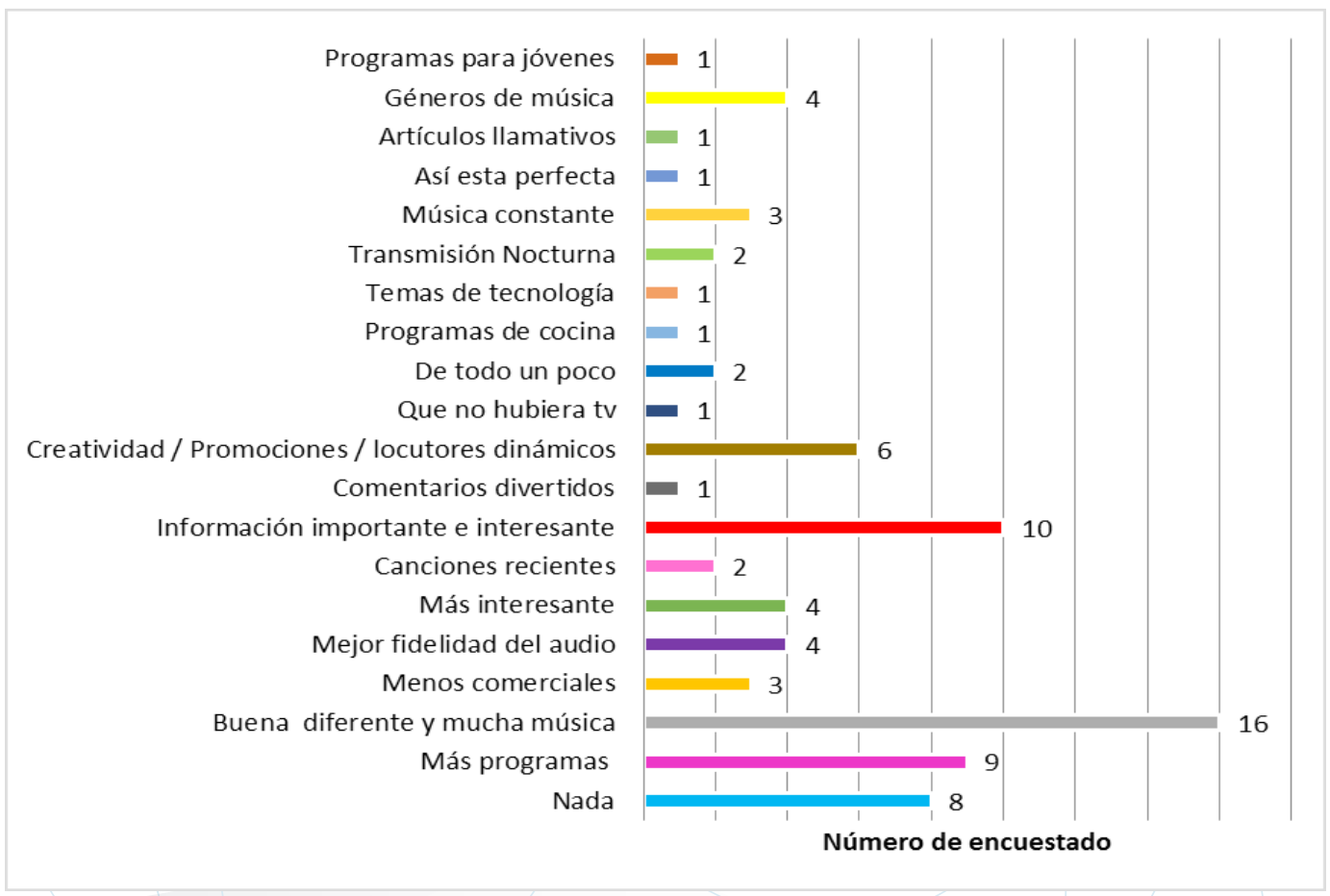

Gráfica 12.- Propuestas de mejora, emitidas por los encuestados. 
En la imagen se aprecian las respuestas al cuestionamiento de los respondientes situados en la zona centro de Acámbaro; sobre ¿qué debería tener la radio para escucharla?; en donde un $20 \%$ (16 encuestados) dijo que fuera buena diferente y mucha música, en cambio el 12.5\% (10 encuestados) respondió información importante e interesante; sin embargo el 11.3\% (9 encuestados) expresó que la radio debería tener más programas; aunado de un 10\% ( 8 encuestados) que emitió la respuesta nada; consideran que lo ofrecido está bien.

Posteriormente se encuentra el $7.5 \%$ (6 encuestados) que dijo creatividad, promociones y locutores dinámicos; por otro lado se encuentran 3 respuestas con 5\% (4 encuestados respectivamente), las cuales son: mejor fidelidad de audio, que sea más interesante y finalmente se concentró en diversos géneros de música, debido a que tuvieron una sola mención trova, rock, música de antes, éxitos de los 80's.

Aunado de los porcentajes anteriores se encuentra el $3.8 \%$ (3 encuestados) con un empate de dos respuestas, una que debe de haber menos comerciales; y otra que recomiendan música constante; así mismo con tendencia del $2.5 \%$ ( 2 encuestados respectivamente) se presentan tres diferentes opiniones:

- Canciones recientes.

- Transmisión nocturna

\section{ANÁLISIS E INTERPRETACIÓN RESULTADOS}

- $\quad$ De todo un poco.

Finalmente con el 1.3\% (1 encuestado respectivamente) se encontraron varias respuestas, las cuales se mencionan a continuación:

- Comentarios divertidos

- Que no hubiera televisión

- Programas de cocina

- Así esta perfecta

- $\quad$ Temas de tecnología

- Artículos llamativos

- $\quad$ Programas para jóvenes

\section{COMPROBACIÓN DE HIPÓTESIS}

La hipótesis planteada se acepta, debido a que la tendencia mostró que la competencia directa de Radio Bajío, es Máxima y EXA; además los datos arrojaron otra estación que no se tenía contemplada y que ofrece algo similar; es Mix 90's, 80's y más y radio Ranchito; esta última es una estación de Morelia, cuyo formato también puede categorizarse como tradicional y antiguo, pero a la gente le agrada.

\section{CONCLUSIÓN Y PROPUESTAS DE MEJORA.}

La aplicación de encuestas fue rica en información, ya que no solo se determinó quién es la competencia directa de la Radio Bajío, si no que desde la culminación de esta primera fase del proyecto; se pueden ir perfilando estrategias mercadológicas; las cuales se retomarán en la fase dos.

Un dato importante que arrojó la investigación fue que pese al avance tecnológico y el internet; la población de Acámbaro aún les agrada escuchar música por la radio; el resultado fue una tendencia favorable de un 64\%; dato que se debe considerar para seguir innovando y que no tenga como consecuencia un declive.

La radio se sigue considerando un medio importante de información; en Acámbaro no se cuenta con un medio que dé a conocer lo que pasa en la ciudad y sus alrededores; por ende, se recomienda que Radio Bajío invierta en realizar reportajes de interés social.

La población acambarense, se inclinó a que no tenían tiempo para escuchar la radio; derivado a eso se propone realizar una campaña, enfocando a este segmento en específico, todo sin perder la esencia de la misma; que se enfoque en que la radio es un medio para estar informado y la cual en cualquier parte la pueden escuchar, paralelo a ello deben intensificar el proceso de comunicación enfocado a que Radio Bajío cuenta con formato en internet; para aquellas personas que no tienen tiempo, se informen y se relajen en cualquier momento del día; con ello se aumentarán los radioescuchas por medio de ese formato; esto debido a que pese al avance que ha tenido la radio con el paso del tiempo, la gente prefiere seguir escuchando en la radio convencional; enfocando a un segmento en específico. Un dato relevante, aunque con una tendencia pequeña de $8 \%$ fue la gente que respondió no tener radio; y el 6\% que dijo no acostumbrarla; la organización radiofónica, al apreciar la directriz de respuestas puede realizar una promoción en la que regale grabadoras y mantengan el regalo de tabletas, esto con el fin de incentivar a las personas a que los escuchen; además de que si se enteran de esta promoción se mantendrán a la expectativa de lo que ofrece la radio.

Se dedujo que la población acambarense es muy pasiva, ya que los programas interactivos no tuvieron 
tendencia positiva, finalmente lo que busca la gente es tener más música y menos comerciales, es por ello que se recomienda que en el segmento que Radio Bajío identifique como adecuado, coloque un programa con una voz atractiva, que bien no hablará demasiado, pero que capte el interés del público; acompaño a su vez con música nueva, ya que se tiene la premisa de que la gente los percibe como anticuados, esto debido a que los locutores ya tienen muchos años al aire. Para lo antes mencionado se necesita realizar un casting de voces nuevas tanto para innovar su programación como para los comerciales, esto no significa que deban correr personal, se pude mantener, y posteriormente realizar fusión en algún programa de gente nueva con el personal que tiene experiencia.

Finalmente se concluye que la competencia de Radio Bajío es Mix 80's, 90's y más

Máxima, EXA y Radio Ranchito; ya que ofertan música de un género muy parecido, este es música pop tanto en inglés como español y; canciones de décadas pasadas; además el análisis arrojó un dato significativo el cual es que un $6 \%$ mencionó no tener preferencia por ninguna $\mathrm{y}$ otro $3 \%$ dijo cualquier estación, es información importante, porque Radio Bajío puede enfocar esfuerzos de su estrategia comercial para que pasen a ser fieles radioescuchas y así se logre cubrir la frase del experto en marketing Philip Kotler "La mejor publicidad es la que hacen los clientes satisfechos" (marketingdirecto. com, 20112011, Recuperado el 17 de abril de 2015), si se concentra en mantener lo anterior podrán aumentar su preferencia en la región, tanto de radioescuchas como de anunciantes.

Los datos anteriormente citados, servirán para comenzar la segunda fase del proyecto.

\section{REFERENCIAS}

- Libros

Alvira, F. (2011). La encuesta: una perspectiva general metodológica. Madrid: Centro de Investigaciones Sociológicas.

Bautista, N. (2011). Proceso de la investigación cualitativa: epistemología, metodología y aplicaciones. Colombia: Editorial El Manual Moderno Colombia.

Equipo Vértice. (2012). Marketing promocional orientado al comercio. España: Vértice.

Garza, A. (2007). Manual de técnicas de investigación para estudiantes de ciencias sociales y humanidades. México: El colegio de México, A. C.

Heinemann, K. (2003). Introducción a la metodología de la investigación empírica en las ciencias del deporte. España: Paidotribo.

Hernández, C. (2011). Planificación y programación. Costa Rica: EUNED.

Hernández, R. (2010). Metodología de la investigación. México: Mc Graw Hill.

Hurtado, L., \& Josefina, T. (2007). Paradigmas y métodos de investigación en tiempos de cambios. Venezuela: CEC, SA.

Kotler, P. (2007). Fundamentos de Marketing. México : Prentice Hall.

Mohammad, N.(2005). Metodología de la investigación. México: Limusa.

Quintana, E., Cabañas, R., \& Gómez, A. (1998). Métodos de investigación. México: Prentice Hall.

Rodríguez, E. (2005). Metodología de la investigación . México: Universidad Juárez Autónama de Tabasco.

Santesmases, M., Sánchez, A., \& Valderrey, F. (2003). Mercadotecnia conceptos y estrategias. Madrid: Pirámide.

Toro, I., \& Parra, R. (2006). Método y conocimiento: metodología de la investigación. Colombia: Fondo EditorialUniversidad EART.

Wells, W., \& Burnett, S. M. (2007). Publicidad principios y prácticas. México: Pearson Prentice Hall.

\section{- $\quad$ Páginas Electrónicas}

ht t p:// c i berconta.unizar.es/leccion/ benchmarketing/200.HTM, Recuperado el 15 de abril de 2015.

http://www3.inegi.org.mx/sistemas/mexicocifras/ default.aspx?e=11, Recuperado el 19 de febrero de 2015.

http://www.marketingdirecto.com/actualidad/ checklists/las-mejores-frases-de-los-grandes-expertosen-marketing/, Recuperado 15 de abril de 2015.

http://www.pdcahome.com/check-list/, Recuperado el 17 de abril de 2015.

http://www.universoformulas.com/estadistica/ inferencia/muestreo-conveniencia/, Recuperado el 17 de abril de 2015 . 
Copyright (c) 2016 M. M. Mayra Verónica Barrera Figueroa

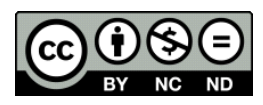

Este texto está protegido por una licencia Creative Commons 4.0.

Usted es libre para Compartir (copiar y redistribuir el material en cualquier medio o formato), siempre que cumpla las condiciones de:

Atribución: Usted debe dar crédito a la obra original de manera adecuada, proporcionar un enlace a la licencia, e in dicar si se han realizado cambios. Puede hacerlo en cualquier forma razonable, pero no de forma tal que sugiera que tiene el apoyo del licenciante o lo recibe por el uso que hace de la obra.

No Comercial: Usted no puede hacer uso de la obra con propósitos comerciales

Sin Derivadas: Si remezcla, transforma o crea a partir de la obra, no podrá distribuir la obra modificada

$\underline{\text { Resumen de la licencia - Texto completo de la licencia }}$ 